Ann. Sci. For., 1984, 41 (3), 323-334

\title{
Influence du froid, de la lumière et de stress hydriques sur la formation du bois de hêtre
}

\author{
Françoise HUBER \\ I.N.R.A., Station de Recherches sur la Qualité des Bois \\ Centre de Recherches forestières \\ Champenoux, F 54280 Seichamps
}

\begin{abstract}
Résumé
L'accroissement radial et la taille des vaisseaux sont étudiés chez de jeunes plants de hêtre en fonction de l'apport en eau, de la lumière qu'ils reçoivent, ainsi qu'en fonction de la température à laquelle ils sont soumis pendant leur dormance.

L'analyse statistique montre l'importance du rôle joué par l'eau et la lumière, ainsi que celui du froid dont l'intensité favorise la croissance radiale.

La taille des vaisseaux est influencée de façon significative par la lumière d'une part, mais surtout par l'effet combiné de la lumière et de la température pendant la phase de repos végétatif. Lorsque la lumière se raréfie, les plus gros vaisseaux sont obtenus chez les plants mis hors gel.
\end{abstract}

\section{Introduction}

L'influence du froid plus ou moins important subi par les feuillus pendant leur dormance, ainsi que celle de stress hydriques se produisant pendant la période de forte activité végétative, sont peu étudiées.

Cependant, des observations faites sur des arbres plus ou moins bien alimentés en eau montrent l'influence de ce facteur sur la croissance en hauteur, et parfois en diamètre (Longman \& Couts, 1974).

$\mathrm{Au}$ cours d'une année humide, le diamètre du tronc chez Fraxinus nigra et Quercus alba augmente trois fois plus qu'au cours d'une année sèche (FrasEr, 1956 ; BogGESS, 1956).

Chez Quercus alba et Fagus grandifolia, la croissance du cerne est liée à la fois au déficit en eau de l'année en cours et à celui de l'année précédente (FritTs, 1962).

Doley \& Leyton (1968), étudiant les effets de stress hydriques en relation avec les régulateurs de croissance sur la formation du xylème chez le frêne montrent que, comme dans le cas des résineux, le manque d'eau réduit l'activité métabolique, ce qui provoque la raréfaction des hydrates de carbone nécessaires au transfert de l'auxine, ainsi qu'à sa synthèse elle-même. 
En plus de l'eau, dont l'effet indirect peut être responsable des modifications de croissance, le rôle du froid est mis en évidence comme facteur pouvant agir sur l'anatomie du xylème. L'effet des hormones, et principalement celui de A.I.A. varie en fonction du froid subi par les plants lors de leur dormance : ainsi, les vaisseaux du frêne sont de plus petite taille quand il ne subit pas de froid avant l'application du traitement hormonal (ODANI, 1980).

Chez cette essence, la levée de dormance s'accompagne d'une augmentation d'acide gibberellique, mais en revanche, la quantité de substance inhibitrice, qui augmente en fonction de l'intensité du froid subie par le plant pendant la dormance, diminue (EAgLe \& Wareing, 1964).

Les expériences dont il est rendu compte ont été entreprises, contrairement à la quasi-totalité des études précédentes, sur un nombre de plants suffisant pour permetre une analyse statistique des résultats. Elles avaient pour but de déterminer le rôle de l'eau pendant la période de végétation sur des plants ayant subi un froid plus ou moins important lors de leur dormance, ainsi que le rôle quantitatif de la lumière sur l'anatomie d'un bois feuillu homogène : le hêtre.

\section{Matériel et méthode}

- Essence : Fagus silvatica.

- L'expérimentation est faite sur des plants de 2 ans. La premièrc année, ils sont élevés en pépinière jusqu'au début de l'hiver, puis mis en pots étanches (fig. 1).

Avant les premières gelées, une moitié des hêtres est mise hors gel (hêtres I), tandis que les autres (hêtres II) restent dehors, subissant les froids hivernaux.

Dès fin février de l'année suivante, les hêtres II rejoignent les hêtres I en serre et, jusqu'à mi-avril, les deux lots subissent le même traitement hydrique (eau en suffisance), thermique (hors gel), et lumineux (lumière naturelle).

A la mi-avril, les différentes modalités de traitements suivants sont appliquées :

- lumière :

- modalité 1 : lumière naturelle ; le temps d'éclairement est celui du jour ;

- modalités 2 et 3 : lumière artificielle, respectivement 5000 et 2500 lux, appliquée pendant 16 heures/24.

- alimentation en eau :

- modalité E1 : pendant 12 semaines, le pot étanche et son plant sont pesés deux fois par semaine. Si une perte d'eau est observée par rapport à la pesée initiale, on ramène le pot à son poids d'origine en ajoutant de l'eau ;

- modalités E2 et E3 : pendant les trois premières semaines, les plants sont soumis au même traitement que ceux du lot E1, puis pendant six semaines :

- les plants du lot E2 reçoivent arbitrairement $10 \mathrm{ml}$ d'eau deux fois par semaine, quelle que soit leur perte en poids,

- les plants E3 cessent d'être arrosés. 


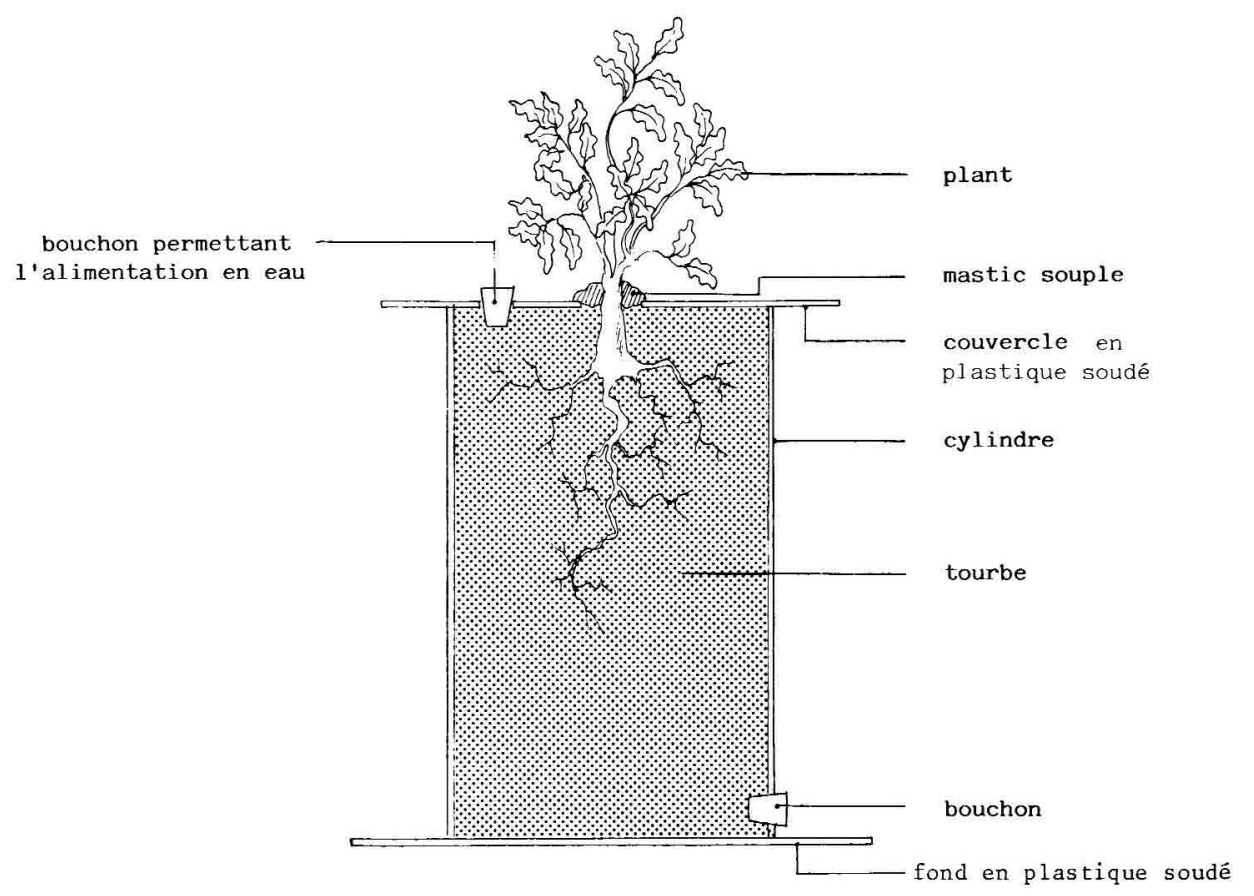

FIG. 1

Schéma d'un pot étanche.

Water-tight pot.

\section{Tableau 1}

Plan de l'expérience.

Experimental drawing.

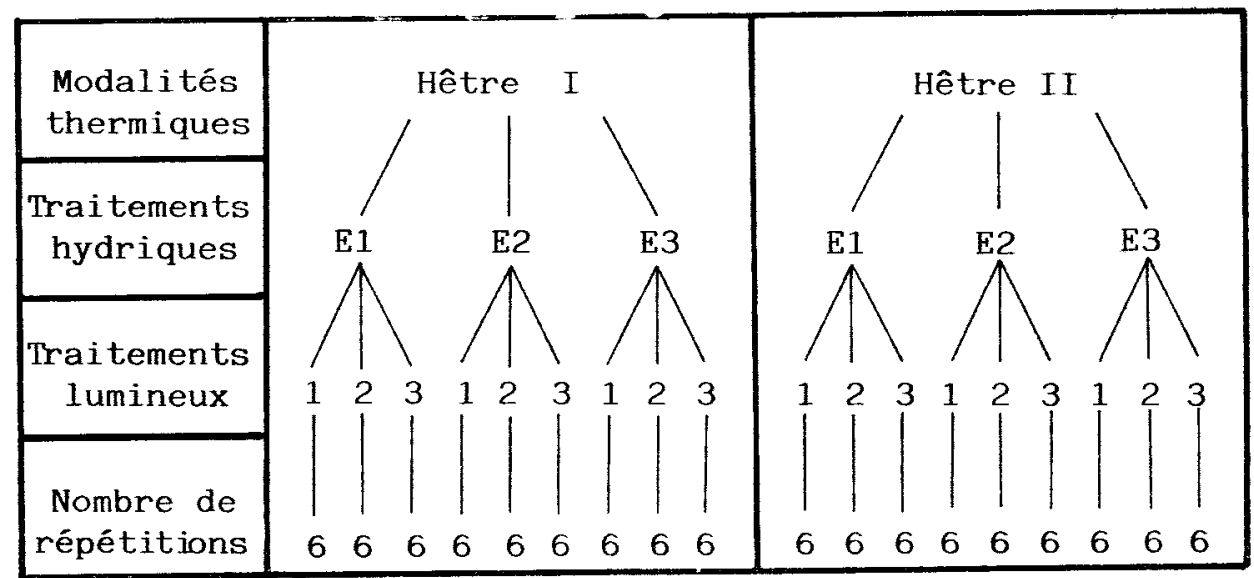


Après neuf semaines de traitement, les lots E2 et E3 sont à nouveau arrosés comme en E1 pendant trois semaines en gardant toujours comme poids de référence celui que les pots avaient au début du traitement.

L'expérience est faite sous des températures diurnes de $20^{\circ} \mathrm{C}$ et nocturnes de $12^{\circ} \mathrm{C}$. Il y a ainsi 18 traitements et 6 répétitions par traitement (tabl. 1).

Après la récolte, des coupes microscopiques transversales sont réalisées sur des fragments de tige prélevés à $2 \mathrm{~cm}$ au-dessus du collet, et colorées à la safranine bleu astra. Sur ces préparations grossies 243 fois par un microscope à projection, on mesure l'accroissement du cerne et la section radiale des vaisseaux d'un secteur choisi au hasard.

\section{— Traitement des données :}

Elles font l'objet d'une analyse de variance orthogonale de modèles factoriels croisés équilibrés ou d'un test $\mathrm{F}$ simple. Lorsque le seuil du F est significatif, les moyennes sont comparées par la méthode de Student (test $t$ corrigé).

Les seuils de signification du test $\mathrm{F}$ sont notés comme suit :

$$
\begin{aligned}
& \text { * } 5 \text { p. } 100 \\
& * \quad 1 \text { p. } 100 \\
& * * * 1 \text { p. } 1000
\end{aligned}
$$

NS non significatif.

Pour le test $\mathbf{t}$, toutes les valeurs figurant sous un même trait ne diffèrent pas de façon significative au scuil de 5 p. 100.

Le code utilisé dans les tableaux et dans le texte comprend un premicr chiffre correspondant au traitement lumincux, puis la lettre $\mathrm{E}$ suivic d'un deuxième chiffre donnant l'indication du traitement hydrique.

\section{Résultats}

\subsection{Accroissement radial}

Cette mesure (tabl. 2) donne une vue d'ensemble de l'activité cambiale, bien qu'elle ne permette pas de préciser, en cas de différence observée cntre traitements, où elle se situe (un accroisscment plus important peut être dû̀ à une plus grande production cellulaire, à de plus grosses cellules ou à une combinaison des deux).

Les différences d'accroissement sont hautement significatives en fonction des traitements (tabl. 3). Cependant, aucun effet de l'interaction eau - lumière, ou lumic̀re - traitement thermique, ne semble influencer cette croissance.

L'analyse des effets principaux montre que :

- tous autres traitements confondus, le hêtre mis en pépinière pendant la période hivernale a une croissance deux fois supérieure à celle des plants mis hors gel $(1,30 \mathrm{~mm}$ contre 0,65$)$. 


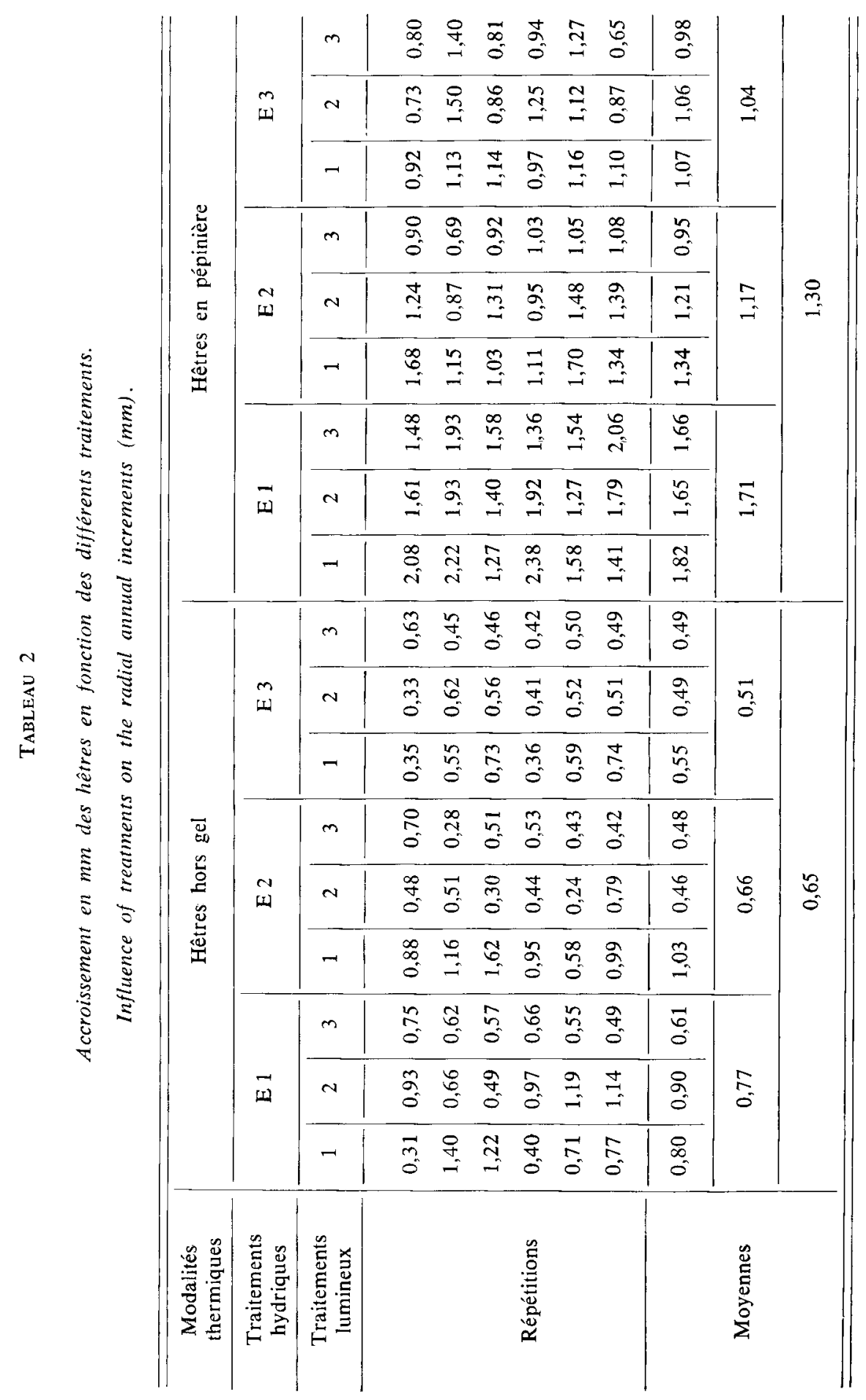


TABLEAU 3

Analyse de variance de l'accroissement annutel radial du hêtre.

Analysis of variance on the annual radial increments of beech.

\begin{tabular}{|c|c|c|c|c|}
\hline Origine de la variation & $\begin{array}{l}\text { Somme } \\
\text { des carrés }\end{array}$ & $\mathrm{ddl}$ & Variance & F calculé \\
\hline Effet thermique (T) & 688482,67 & 1 & 688482,67 & $173,9 * * *$ \\
\hline Effet hydrique (E) . & $244.520,38$ & 2 & 122260.19 & $30,89 * * *$ \\
\hline Effet lumineux (L). & 62767,05 & 2 & 31383,52 & $7,93 * * *$ \\
\hline Interaction $\mathrm{T} \times \mathrm{E}$. & 65227.01 & 2 & 32613,50 & $8,24 * * *$ \\
\hline Interaction $\mathrm{E} \times \mathrm{L} \ldots$ & 36739.55 & 4 & 9184.88 & $2,32 \mathrm{NS}$ \\
\hline Interaction $\mathrm{T} \times \mathrm{L} \ldots \ldots \ldots$ & 1594.01 & 2 & 797,00 & $0,20 \mathrm{NS}$ \\
\hline Interaction $\mathrm{T} \times \mathbf{E} \times \mathrm{L} \ldots \ldots$ & 378879.21 & 4 & 94719,80 & $23,93 * * *$ \\
\hline Erreur $\ldots \ldots \ldots \ldots \ldots \ldots$ & 3562,37 & 90 & 3958,19 & \\
\hline To:al & 1481772,25 & 107 & & \\
\hline
\end{tabular}

TABLEAU 4

Comparaison de moyennes de l'accroissement radial en fonction des traitements hydriques.

Comparison of means on the annual radial increments between the hydric treaments.

\begin{tabular}{l|c|c|c|c|}
\hline Effet hydrique $\ldots \ldots \ldots \ldots \ldots \ldots \ldots \ldots \ldots \ldots$ & E 1 & E 2 & E 3 \\
\hline Accroissement radial moyen en $\mathrm{mm} \ldots \ldots \ldots \ldots$ & 1.24 & 0,91 & 0,77 \\
\hline
\end{tabular}

\section{TABleau 5}

Comparaison de moyennes de l'accroissement radial en fonction des traitements lamineux.

Comparison of means of the annual radial increments between the different light-intensity treatments.

\begin{tabular}{|c|c|c|c|}
\hline Effet lumière & 1 & 2 & 3 \\
\hline Accroissement radial moyen en $\mathrm{mm}$ & 1,11 & 0,96 & 0,86 \\
\hline
\end{tabular}




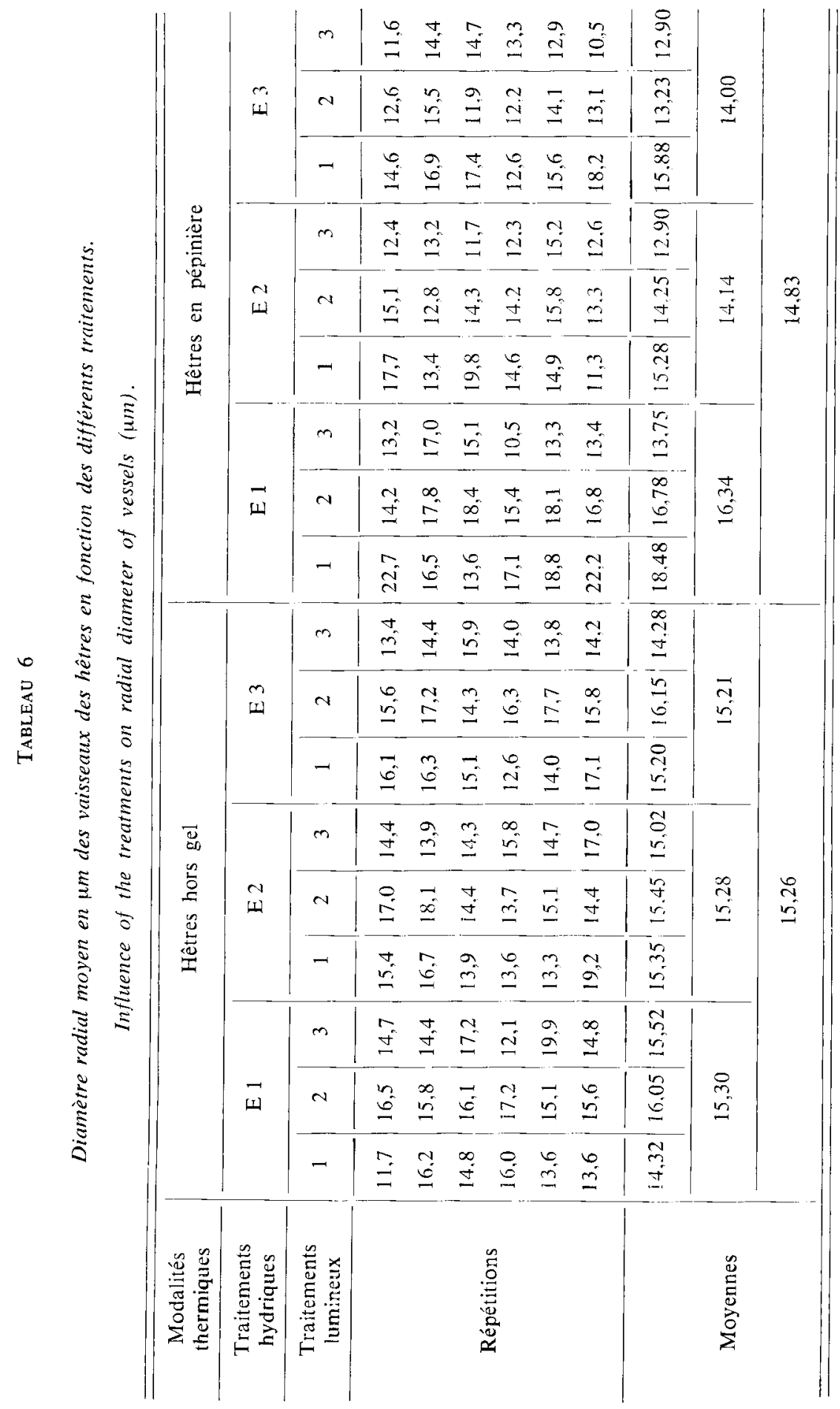


Cette différence demeure si on ne lient compte que de l'effet thermique, c'est-àdire si on compare l'accroissement des plants dans le traitement où cau et lumière sont en suffisance (traitement 1E1).

L'accroissement radial diffère de façon significative $\left(\mathrm{F}_{5}^{1}=6,61 *\right.$ ), mais le seuil est moins élevé, montrant que la croissance est certes liée au froid, mais aussi aux traitements subis par ces derniers au cours de la période de végétation.

- Quelle que soit la modalité thermique, la croissance est plus importante avec un bon apport d'eau (tabl. 4).

- Une différence de croissance en fonction du traitement lumineux n'apparaît qu'entre la lumière naturelle et 2500 lux (tabl. 5).

\subsection{Diamètre radial des vaisseaux (tabl, 6)}

La lumière est le facteur le plus important des trois principaux effets (tabl. 7). Les vaisseaux de plus grand diamètre sont obtenus soit en lumière naturelle, soit sous 5000 lux (tabl. 8).

\section{Tableav 7}

Analyse de variance sur le diamètre radial des vaisseaux.

Analysis of variance on the radial diameter of vessels.

\begin{tabular}{|c|c|c|c|c|}
\hline Origine de la variation & $\begin{array}{c}\text { Somme } \\
\text { dzs carrés }\end{array}$ & ddl & Variance & F calculé \\
\hline Effet thermique (T) & 0,3267 & 1 & 0.3267 & $1,50 \mathrm{NS}$ \\
\hline Effet hydrique (E) .. & 1.9299 & 2 & 0,9649 & $4,44 *$ \\
\hline Effet lumineux (L) .. & 3.2513 & 2 & 1.6256 & $7,49 * * *$ \\
\hline Interaction $\mathrm{T} \times \mathrm{E} \ldots \ldots \ldots \ldots$ & 1,7610 & 2 & 0,8805 & $4,06 *$ \\
\hline Interaction $E \times L \ldots \ldots \ldots \ldots$ & 0,2579 & 4 & 0,6449 & $2.97 *$ \\
\hline Irteraction $\mathrm{T} \times \mathrm{L} \ldots \ldots \ldots \ldots$ & 3,4142 & 2 & 1.7071 & $7,86 * * *$ \\
\hline Interaction $\mathrm{T} \times \mathbf{E} \times \mathbf{L} \ldots \ldots$ & 1.2645 & 4 & 0,3161 & $1,46 \mathrm{NS}$ \\
\hline Erreur & 19.5429 & 90 & 0.2171 & \\
\hline Total & 31,7484 & 107 & & \\
\hline
\end{tabular}

\section{Tableav 8}

Comparaison de moyennes du diamère radial des vaisseaux de hêtre - traitement lumière. Comparison of means on the radial diameter of vessels - light intensity treatments.

\begin{tabular}{l|c|c|c|c}
\hline Effet lumière ............................... & 1 & 2 & 3 \\
\hline Diamètre radial moyen des vaisseaux en $\mathrm{mm} \ldots$ & 0.0157 & 0,0153 & 0,0141 \\
\hline
\end{tabular}


L'analyse de variance met en relief l'importance de l'eau, et c'est avec le meilleur apport d'eau que sont obtenus les vaisseaux les plus gros (tabl. 9).

L'effet thermique seul paraît sans influence sur leur taille. En revanche, il faut remarquer la valeur très élevée du $\mathrm{F}$ donnée par l'analyse de l'interaction de l'effet thermique et lumineux (tabl. 7). L'importance de ce dernier effet ayant été montré à l'intérieur de chaque régime lumineux, les modalités thermiques sont étudiées.

L'analyse de variance (tabl. 10) montre que cet effet est d'autant plus ressenti que les plants sont moins éclairés.

\section{Tableau 9}

Comparaison de moyennes du diamètre radial des vaisseaux du hêtre - traitement hydrique. Comparison of means on the radial diameter of vessels - hydric treatments.

\begin{tabular}{l|c|c|c}
\hline Effet eau $\ldots \ldots \ldots \ldots \ldots \ldots \ldots \ldots \ldots \ldots \ldots \ldots$ & E 1 & E 2 & E 3 \\
\hline Diamètre radial moyen des vaisseaux en mm .... & $\underline{0,0158}$ & 0,0147 & 0.0146 \\
\hline
\end{tabular}

TABLeaU 10

Analyse de variance comparant l'effet thermique en fonction de chaque traitement lumineux. Analysis of variance on the thermic effects to the each light intensity treatment.

\begin{tabular}{|c|c|c|}
\hline Traitement lumineux & $F_{31}^{1}$ calculé & Seuil de signification \\
\hline Lumière naturelle & 3.51 & NS \\
\hline $5000 \operatorname{lux}$ & 4,35 & * \\
\hline $2500 \operatorname{lux}$ & 9,56 & $\because *$ \\
\hline
\end{tabular}

Dans le cas où des différences entre les traitements thermiques apparaissent, ce sont les plants soumis au froid hivernal qui ont les plus petits vaisseaux : sous 5000 lux, ils mesurent 14,8 $\mathrm{um}$ contre 15,9, et 13,2 um contre 14,9 sous 2500 lux.

\section{Conclusion}

Ces travaux montrent l'importance du froid subi par les plants pendant leur dormance sur la structure du bois mise en place pendant la saison de végétation suivante. 
En absence de gel, les plants ont une croissance en diamètre plus faible. Cette différence peut être attribucé à une formation moindre de cellules liće à une concentration en acide gibberellique plus faible chez les végétaux n'ayant pas subi de basses températures en hiver (EAgles \& WAREING, 1963). Cette hormone, outre son importance dans la croissance en hauteur (SAchs et al., 1959), a un rôle non moindre dans la division cellulaire au niveau cambial qu'elle favorise (DIBGY \& WAREING, 1966).

La croissance, dans les limites fixées dans l'expérience, est peu sensible à l'intensité lumineuse ; cependant, la comparaison de moyennes montre qu'il y a un seuil au-dessous duquel l'arbre manifeste une réaction : il se situe entre 5000 et 2500 lux, compte tenu des modalités fixées pour les autres traitements.

Concernant la formation des vaisseaux, on peut admettre que sous l'effet de la lumière, il y a synthèse de substances responsables, non plus de la multiplication cellulaire, mais de la plasticité des membranes, donc de la taille des cellules, substances qui agiraient en interaction avec les "hormones " formées en hiver.

Dans le cas où la lumière est en suffisance, cette substance, formée au printemps, serait abondante; elle se répartirait dans l'ensemble des cellules quel que soit leur nombre et masquerait les effets antagonistes de celles formées pendant la dormance.

Dans le cas contraire, et chez les plants restés dehors en hiver, il y aurait une forte activité cellulaire (accroissement radial important) liée à la présence d'acide gibberellique,mais la substance formée au printemps serait, par manque de lumière, peu abondante et devrait être distribuée à un nombre de cellules plus grand que chez les plants restés hors gel, d'où la taille moindre des vaisseaux. Cet effet ne s'est peutêtre pas limité à ces derniers.

\section{Remerciements}

Ces travaux ont été réalisés grâce à l'aide financière de la D.G.R.S.T., et technique de $M^{\text {me }}$ Garros que je tiens à remercier.

\section{Summary}

The effects of chilling, light intensity and hydric stress on wood formation of beech

The cambial activity is favoured by watering and light intensity during the growth period, and chilling during the dormance.

The vessels size is influenced by the light intensity, there is also an interaction between light and chilling. vessels.

When the light intensity is low, the plants, having not been exposed, produce the larger

Reçu le 26 novembre 1982.

Accepté le 29 juillet 1983. 


\section{Références bibliographiques}

BogGess W.R., 1956. Weckly diameter growth of shortleaf pine and white oak as related to soil moisture. Proc. Soc. Am. Foresters, 83-89.

Digby J., Wareing P.F., 1966. The effect of applied growth hormones on cambial division and the differential of the cambial derivatives. Ann. Bst,, N.S., vol. 30, n" 119.

Doley D., Leyton L., 1968. Effects of growth regulating substances and water potential on the development of secondary xylem in Fraxinus. New Phytol., 67, 579-594.

Eagles C.F., Wareing P.F. ,1963. Dormancy regulators in woody plants. Nature, Lond., $199,874$.

Eagles C.F., Wareing P.F., 1964. The role of growth substances in the regulation of bud dormancy. Physiol. Plan., 17, 697-709.

Fraser D.A., 1956. Ecological studies of forest trees at Chalk River, Ontario, Canada. II - Ecological conditions and radial increment. Ecology, 37, 777-789.

Fritrs H.C., 1962. The relation of growth ring widths in American beech and white oak to variation in climate. Tree Ring Bulletin, 25, 2-10.

Longman K.A., Couts M.P., 1974. Physiology of the oak tree. In : The British Oak (H.G. Morris and F.H. Perrings ed), E.W. Classey Ltd, Berkshi.

ODANI K., 1980. Effects of chilling of exogenous Indole 3-acetic-acid-mediated wood formation and vessel diameter of white ash and poplar. Mokuzai Gakkaishi, vol. 26, $n^{\circ} 3,127-131$.

SACHS R.M., BREtz C.F., LANG A., 1959. Shoot histogenesis : the carly effects of gibberellin upon stem elongation in two rosette plants. Am. J. Bot., 46, 376-384. 